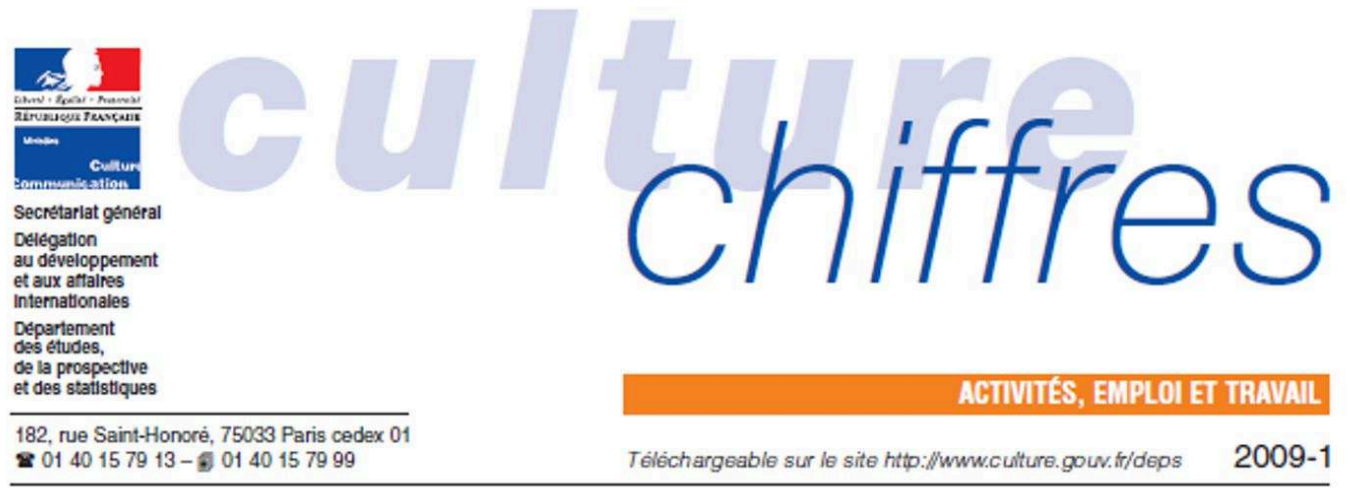

\title{
L'emploi salarié dans le secteur de la culture
}

Éric Cléron, Frédérique Patureau*

Le salariat représente les deux tiers de l'emploi dans le champ des activités culturelles. Il se caractérise globalement, au-delà des différences propres à chaque domaine particulier d'activités, par une très grande hétérogénéité des volumes individuels de travail et de revenu, par un recours fréquent des salariés à d'autres activités extérieures au champ culturel et, surtout, par une instabilité de l'emploi que révèlent simultanément l'importance du CDD et du temps partiel, mais aussi l'ampleur de la rotation (turn-over) des individus d'une année sur l'autre.

En 2006, 770000 individus ont été salariés dans les établissements du secteur culturel ${ }^{1}$. Le spectacle vivant et l'audiovisuel concentrent à eux seuls les deux tiers de ces salariés : un tiers sont salariés d'établissements du spectacle vivant $(34 \%), 16 \%$ d'établissements du cinéma et de la vidéo et $15 \%$ d'établissements de la radio et télévision. Les autres secteurs représentent une part beaucoup plus modeste (de $4 \%$ à $14 \%$ ) de l'emploi salarié du champ culturel (graphique 1).

Une durée annuelle moyenne de travail plutôt faible

Le volume total d'emploi salarié dans le champ des activités culturelles représente environ 530 millions d'heures de travail, soit l'équivalent de 332000 emplois à temps complet ${ }^{2}$. Rapproché de l'effectif salarié total $(770000)$, ce chiffre signale d'emblée une première
Graphique 1-Répartition des salariés selon le secteur d'activité en 2006

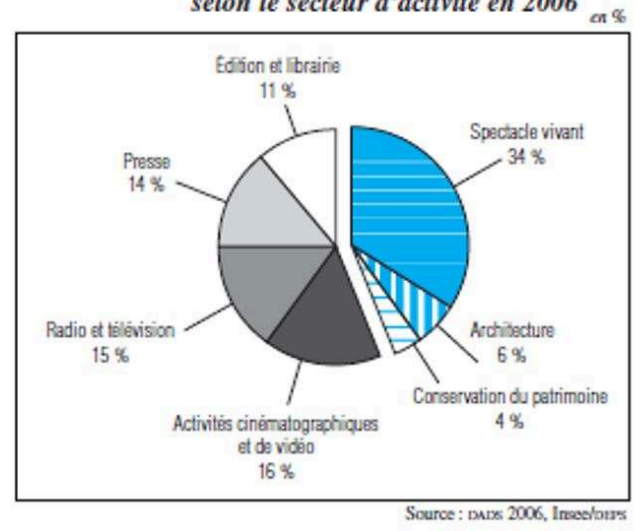

caractéristique du salariat du secteur: la présence d'un très grand nombre de salariés ayant effectué, dans l'année, des périodes de travail extrêmement courtes.

De fait, la durée annuelle moyenne de travail d'un salarié de la culture est de 744 heures (moins de l'équivalent d'un mi-temps). La moitié des salariés a travaillé moins de 406 heures dans l'année et seulement $23 \%$ ont travaillé l'équivalent d'un temps complet.

Ces disparités traduisent directement une très forte hétérogénéité des périodes de travail selon le secteur d'activité (tableau 1).

* Éric Cléron est attaché statisticien de l'Insee, Frédérique Patureau est chargée d'études au nurs.

1. Ces données sont issues d'une exploitation statistique des Déclarations annuelles de données sociales (Drns) 2006. Sont considérés ici comme salariés des établissements du secteur culturel tous les individus ayant travaillé au moins une heure au cours de l'année 2006 dans un établissement de ce secteur (voir encadré méthodologique p. 8).

2. Un temps complet représente environ 1650 heures annuelles de travail (woir Magali Bøwr, $\propto$ Les salariés à temps complet travaillent 1650 heures 2. Un temps complet représente environ 1650 heures an 


\section{L'emploi salarié dans le secteur de la culture}

\section{Eric Cléron et Frédérique Patureau}

Éditeur : Département des études, de la prospective et des statistiques

Lieu d'édition : Paris

Année d'édition : 2009

Date de mise en ligne : 21 septembre 2015

Collection : Culture chiffres

ISBN électronique : 9782111398429

\section{Sbooks}

http://books.openedition.org

\section{Édition imprimée}

Date de publication : 1 février 2009

Nombre de pages : 8

Référence électronique

CLÉRON, Eric ; PATUREAU, Frédérique. L'emploi salarié dans le secteur de la culture. Nouvelle édition [en ligne]. Paris : Département des études, de la prospective et des statistiques, 2009 (généré le 25 avril 2021). Disponible sur Internet : <http://books.openedition.org/deps/599>. ISBN : 9782111398429. 

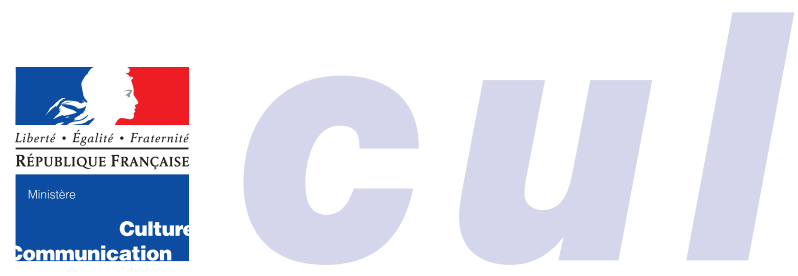

Secrétariat général

Délégation

au développement

et aux affaires

internationales

Département

des études,

de la prospective

et des statistiques

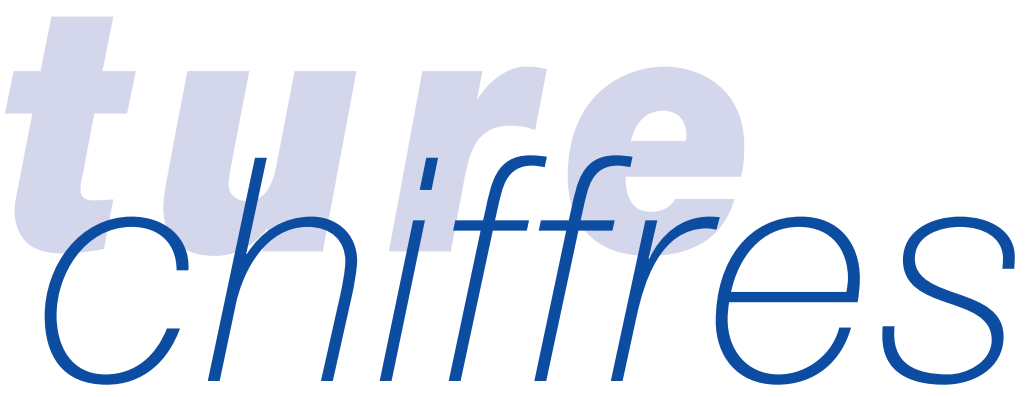

\section{L'emploi salarié dans le secteur de la culture}

\section{Éric Cléron, Frédérique Patureau*}

Le salariat représente les deux tiers de l'emploi dans le champ des activités culturelles. Il se caractérise globalement, au-delà des différences propres à chaque domaine particulier d'activités, par une très grande hétérogénéité des volumes individuels de travail et de revenu, par un recours fréquent des salariés à d'autres activités extérieures au champ culturel et, surtout, par une instabilité de l'emploi que révèlent simultanément l'importance du CDD et du temps partiel, mais aussi l'ampleur de la rotation (turn-over) des individus d'une année sur l'autre.

En 2006, 770000 individus ont été salariés dans les établissements du secteur culturel ${ }^{1}$. Le spectacle vivant et l'audiovisuel concentrent à eux seuls les deux tiers de ces salariés : un tiers sont salariés d'établissements du spectacle vivant (34\%), $16 \%$ d'établissements du cinéma et de la vidéo et $15 \%$ d'établissements de la radio et télévision. Les autres secteurs représentent une part beaucoup plus modeste (de $4 \%$ à $14 \%$ ) de l'emploi salarié du champ culturel (graphique 1).

\section{Une durée annuelle moyenne de travail plutôt faible}

Le volume total d'emploi salarié dans le champ des activités culturelles représente environ 530 millions d'heures de travail, soit l'équivalent de 332000 emplois à temps complet ${ }^{2}$. Rapproché de l'effectif salarié total (770 000), ce chiffre signale d'emblée une première
Graphique 1 - Répartition des salariés selon le secteur d'activité en 2006

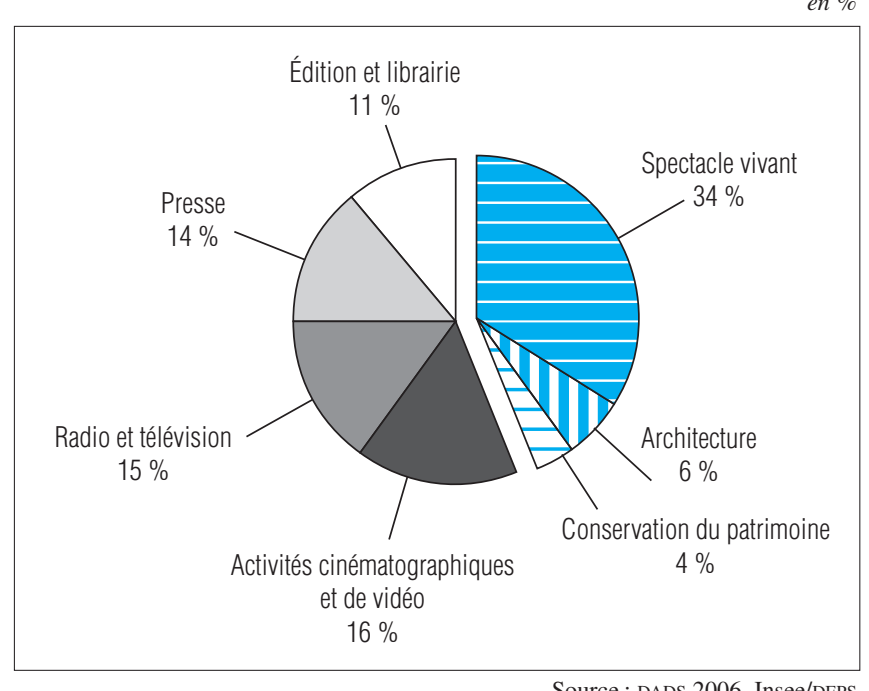

Source : DADS 2006, Insee/DEPS

caractéristique du salariat du secteur: la présence d'un très grand nombre de salariés ayant effectué, dans l'année, des périodes de travail extrêmement courtes.

De fait, la durée annuelle moyenne de travail d'un salarié de la culture est de 744 heures (moins de l'équivalent d'un mi-temps). La moitié des salariés a travaillé moins de 406 heures dans l'année et seulement $23 \%$ ont travaillé l'équivalent d'un temps complet.

Ces disparités traduisent directement une très forte hétérogénéité des périodes de travail selon le secteur d'activité (tableau 1).

\footnotetext{
* Éric Cléron est attaché statisticien de l'Insee, Frédérique Patureau est chargée d'études au DEPS.

1. Ces données sont issues d'une exploitation statistique des Déclarations annuelles de données sociales (DADS) 2006. Sont considérés ici comme salariés des établissements du secteur culturel tous les individus ayant travaillé au moins une heure au cours de l'année 2006 dans un établissement de ce secteur (voir encadré méthodologique p. 8).

2. Un temps complet représente environ 1650 heures annuelles de travail (voir Magali BEFFY, «Les salariés à temps complet travaillent 1650 heures dans l'année », Insee Première, $n^{\circ}$ 1066, février 2006).
} 


\section{Spectacle et audiovisuel : des durées de travail faibles, une grande instabilité de l'emploi}

Dans les activités du spectacle et de l'audiovisuel (cinéma et vidéo, radio et télévision, spectacle vivant), ces durées annuelles sont globalement faibles - qu'elles soient exprimées par des durées moyennes ou médianes ${ }^{3}$ : - les salariés du cinéma et de la vidéo travaillent ainsi en moyenne 471 heures et un salarié sur deux, moins de 104 heures dans l'année ;

- les salariés du spectacle vivant travaillent 412 heures et un salarié sur deux, moins de 125 heures.

Ces activités sont aussi celles où la part des CDI et des temps complets est la plus faible : seulement $22 \%$ de CDI et $40 \%$ de temps complets dans le spectacle vivant, $24 \%$ de CDI et $50 \%$ de temps complets dans les activités cinématographiques et de vidéo.

\section{Architecture, conservation du patrimoine, édition et librairie, presse : des conditions d'emploi plus favorables aux salariés}

Un second sous-ensemble regroupant les autres activités culturelles (hors spectacle et audiovisuel) offre à ses salariés des durées annuelles de travail beaucoup plus conséquentes et a plus généralement recours au CDI et au temps complet. Dans l'architecture, notamment, les trois quarts des salariés sont employés en CDI, et la même proportion a travaillé à temps complet.

$\mathrm{Au}$ sein de cet ensemble, les activités du patrimoine se distinguent par un recours modéré au CDI (38\% contre respectivement $68 \%$ et $64 \%$ dans l'édition et librairie et dans la presse). Plus précisément, le patrimoine est (avec la presse) le secteur où la part de salariés employés sur d'autres types de contrats que le CDI et le CDD est la plus importante $(17 \%)$. Il s'agit, notamment, des diffé- rentes formes de contrats aidés (contrat d'accompagnement dans l'emploi CAE, contrat emploi-jeune CEJ, contrat emploi-solidarité CES, contrat nouvelle embauche CNE...).

La comparaison des durées de travail moyennes et médianes pour chacun des secteurs signale en outre une autre différence importante entre les deux sousensembles: dans les activités du spectacle et de l'audiovisuel, l'écart très marqué entre ces deux valeurs, qui atteint son maximum dans les activités de radio et télévision (écart de 1 à 3 ), traduit une dispersion particulièrement forte des temps de travail des salariés. Jointe aux autres facteurs de fragilisation de l'emploi déjà cités (durées annuelles de travail faibles, flexibilité de la relation d'emploi par recours au CDD, part importante des postes à temps partiel), cette hétérogénéité caractérise un ensemble d'activités que l'on sait marquées par le recours à l'intermittence et par une répartition inégalitaire du travail et de la réussite professionnelle.

\section{Huit salariés sur dix ont travaillé dans un seul établissement en 2006}

Une très forte majorité des salariés de la culture a travaillé dans un seul établissement ${ }^{4}$ au cours de l'année. Cette caractéristique s'observe dans toutes les activités, y compris celles où l'instabilité statutaire de l'emploi est la plus marquée - le spectacle vivant notamment, où plus des trois quarts des salariés sont dans ce cas (tableau 2).

Les secteurs où s'observent les durées annuelles de travail les plus faibles et une généralisation des CDD et temps partiels sont aussi ceux dans lesquels la part des salariés ayant travaillé dans plusieurs établissements différents est la plus élevée : un salarié sur cinq dans les activités cinématographiques et de vidéo, dans celles de la radio et télévision et dans celles du spectacle vivant.

\section{Tableau 1 - Les grandes caractéristiques du travail selon le secteur d'activité en 2006}

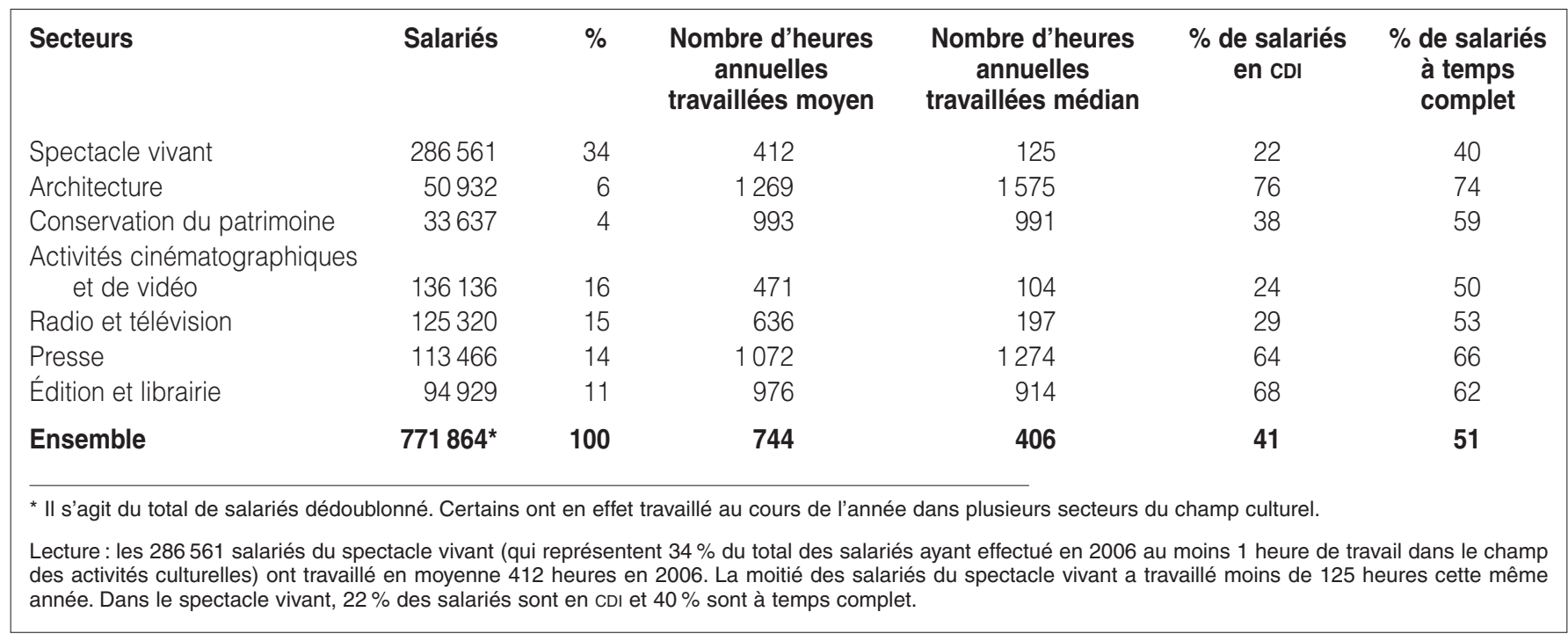

3. La durée médiane partage la population en deux sous-populations égales, celle qui travaille moins que cette durée médiane, et celle qui travaille plus que cette durée.

4. Voir la description des établissements de la culture, encadré p. 8. 
Tableau 2 - Répartition des salariés selon le nombre d'établissements et le nombre de périodes de travail en 2006

\begin{tabular}{|c|c|c|c|c|c|c|}
\hline & \multicolumn{3}{|c|}{ Nombre d'établissements } & \multicolumn{3}{|c|}{ Nombre de périodes } \\
\hline & 1 & 2 & 3 ou + & 1 & 2 & 3 ou + \\
\hline Spectacle vivant & 77 & 11 & 12 & 67 & 13 & 20 \\
\hline Architecture & 95 & 5 & $\varepsilon$ & 89 & 9 & 2 \\
\hline Conservation du patrimoine & 98 & 2 & $\varepsilon$ & 79 & 14 & 7 \\
\hline Activités cinématographiques et de vidéo & 81 & 9 & 10 & 73 & 12 & 15 \\
\hline Radio et télévision & 81 & 10 & 9 & 70 & 13 & 17 \\
\hline Presse & 88 & 7 & 5 & 78 & 12 & 10 \\
\hline Édition et librairie & 95 & 4 & 1 & 87 & 9 & 4 \\
\hline Ensemble & 82 & 8 & 10 & 74 & 12 & 14 \\
\hline
\end{tabular}

Cette dispersion de l'emploi sur plusieurs employeurs s'accompagne d'un plus grand nombre de périodes de travail $^{5}$ : $20 \%$ des salariés du spectacle vivant et $17 \%$ de ceux de la radio et télévision ont eu trois périodes d'emploi (ou plus) au cours de l'année, alors que seulement $2 \%$ des salariés de l'architecture et $4 \%$ de ceux des activités d'édition et librairie sont dans ce cas.

\section{Graphique 2 - Nombre d'heures annuelles de travail médian selon le nombre de périodes de travail en 2006, par secteur d'activité}

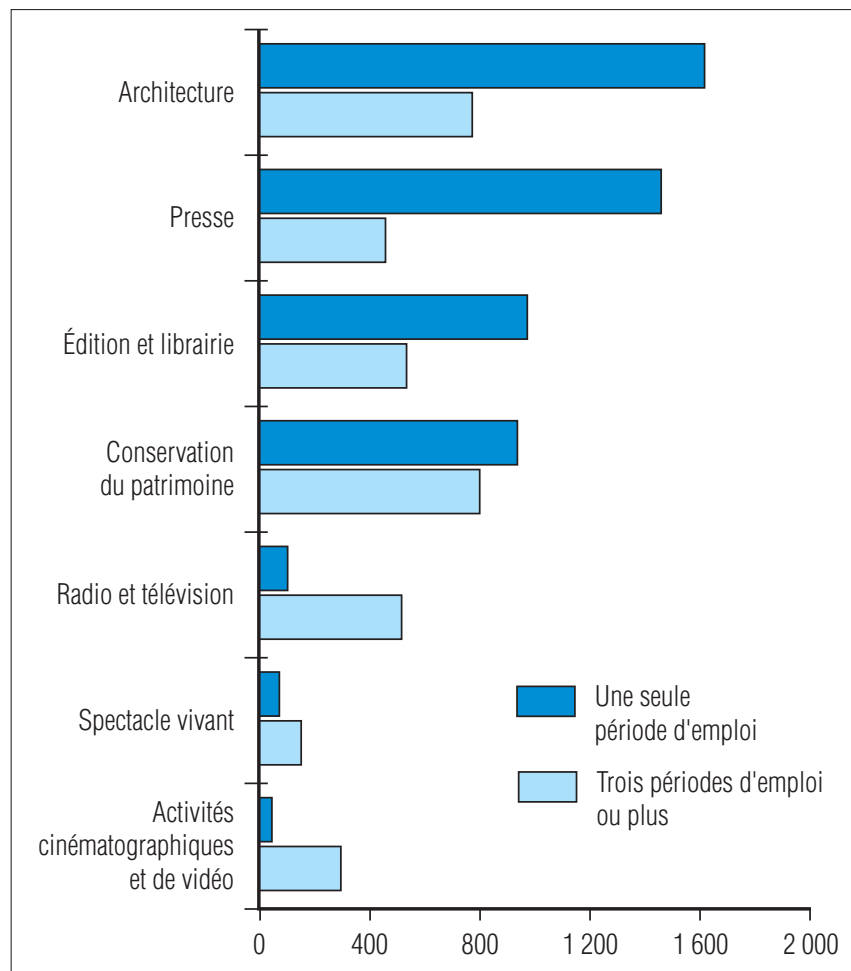

Lecture: la moitié des salariés de l'architecture n'ayant eu qu'une seule période d'emploi en 2006 a travaillé plus de 1600 heures dans l'année.
Enfin, le cumul des établissements et des périodes de travail ne revêt pas la même signification selon le secteur:

- dans les secteurs du spectacle - et à la notable exception des salariés travaillant l'équivalent d'un temps complet -, il s'accompagne d'un volume de travail médian sensiblement plus élevé que celui observé pour les salariés travaillant dans un seul établissement ou ayant une seule période d'emploi (graphique 2). L'observation corrobore pleinement les analyses qui ont démontré les effets positifs de la diversification du portefeuille d'employeurs sur le volume global d'emploi des salariés, sur les marchés du travail fortement dominés par le recours au CDD intermittent;

- dans les autres secteurs (architecture, presse, édition et librairie, conservation du patrimoine), la dispersion de l'activité est, à l'inverse, le propre des salariés ayant de faibles volumes de travail sur l'année, donc peu insérés dans le secteur.

\section{Un salarié sur quatre a également travaillé en dehors du secteur culturel}

En 2006, $28 \%$ des salariés de la culture ${ }^{6}$ ont travaillé en dehors du secteur culturel (graphique 3). Ces salariés ont effectué en moyenne 508 heures dans ces activités extérieures (tableau 3).

Cette spécificité, qui est à rapprocher du faible temps de travail annuel moyen effectué dans les établissements du secteur culturel, mérite d'autant plus d'être soulignée qu'elle s'observe dans tous les secteurs : à l'exception de l'architecture où la part des salariés ayant travaillé hors champ est inférieure à $20 \%$ (et où, par ailleurs, les durées moyennes de travail à l'intérieur du champ culturel sont les plus élevées), le recours à une activité salariée hors champ concerne au minimum un salarié sur cinq (et jusqu'à un salarié sur trois) dans toutes les autres activités (37\% dans les activités de spectacle vivant,

Source : DADS 2006, Insee/DEPS

5. La « période» peut être approximativement assimilée à un contrat (voir la définition dans l'encadré p. 8).

6. Voir le portrait sociodémographique des salariés de la culture, p. 8. 


\section{Graphique 3 - Part des salariés ayant travaillé hors du secteur culturel selon l'activité en 2006}

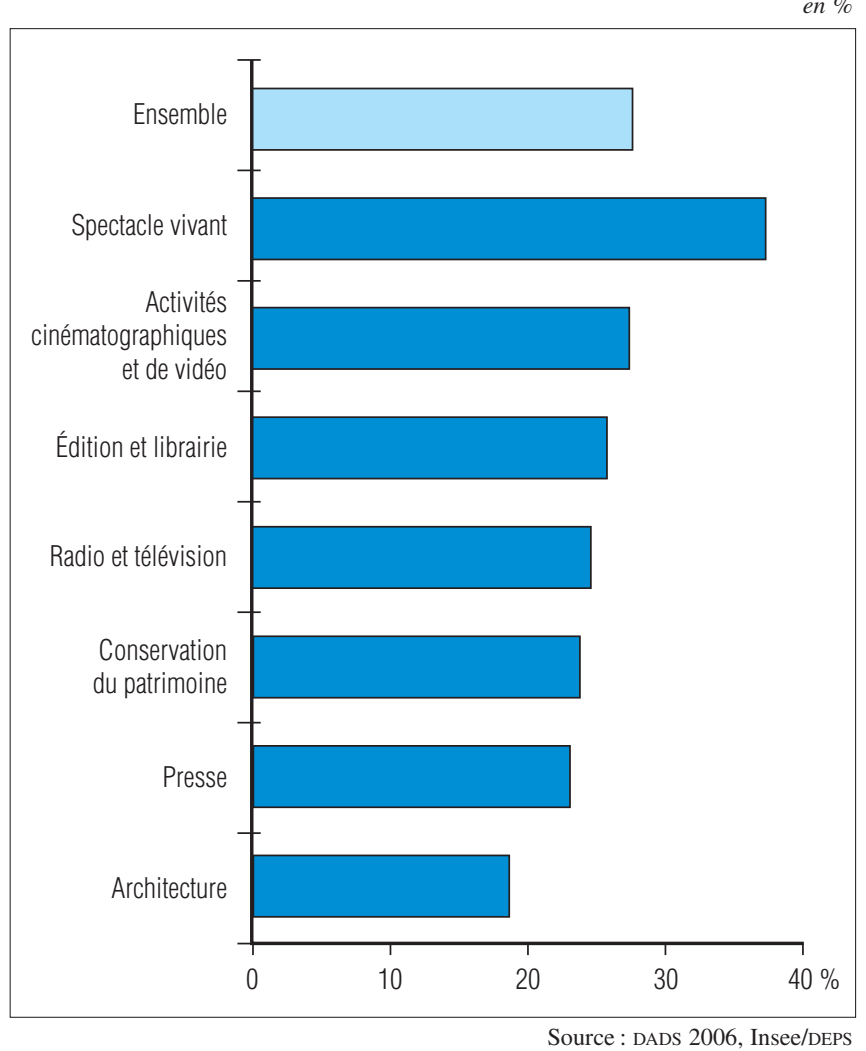

$26 \%$ dans celles d'édition et librairie, $25 \%$ dans la radio et télévision, par exemple).

Plus encore, les salariés concernés par une activité extérieure y effectuent en moyenne des durées de travail supérieures à celles qu'ils effectuent à l'intérieur du champ culturel. L'écart est particulièrement sensible dans les activités du spectacle vivant, du cinéma et de la vidéo où le volume horaire effectué à l'extérieur du champ culturel est en moyenne deux fois plus important que celui réalisé à l'intérieur de celui-ci (tableau 3).
L'examen des secteurs d'activité dans lesquels s'investissent les salariés de la culture à l'extérieur de ce champ permet cependant d'avancer que cette diversification s'effectue, pour partie, dans des secteurs d'activité connexes.

C'est le cas, en particulier, de l'architecture, car les secteurs dans lesquels se déploie l'activité hors champ des salariés sont avant tout des secteurs du bâtiment et de la construction, très complémentaires du cœur de compétences des activités d'architecture : secteurs de l'ingénierie et études techniques et de l'administration d'immeubles résidentiels notamment.

Pour tous les autres secteurs d'activité du champ culturel, ce sont les activités d'administration publique générale qui occupent la première ou la seconde place (en termes de volume horaire de travail) dans l'activité hors champ des salariés. Tout particulièrement, ce sont les salariés du spectacle vivant et des activités de l'audiovisuel qui exercent le plus souvent une activité relevant de ce secteur à l'intitulé très générique qui recouvre, notamment, les activités des établissements publics d'enseignement artistique et culturel. Le secteur des activités associatives, également souvent associé, peut sans doute recouvrir ces activités d'enseignement et d'animation qui prolongent classiquement l'activité des professionnels du spectacle.

\section{Des salaires moyens peu élevés et très inégalement répartis}

Les salaires annuels moyens sont faibles, les variations observées d'une activité à l'autre (moins de 6000 euros annuels dans le spectacle vivant, mais plus de 19000 euros dans la presse) reflétant avant tout les variations sectorielles du volume annuel de travail moyen (tableau 4).

Les secteurs où s'observe la plus importante dispersion des durées annuelles de travail sont aussi ceux qui connaissent la répartition la plus inégalitaire des revenus. L'écart entre revenu moyen et revenu médian atteint

Tableau 3 - La diversification de l'activité à l'extérieur du secteur de la culture en 2006

\begin{tabular}{|c|c|c|}
\hline \multirow[t]{2}{*}{ Secteurs } & \multicolumn{2}{|c|}{ Salariés ayant travaillé en dehors du secteur de la culture } \\
\hline & $\begin{array}{l}\text { Nombre d'heures moyen hors champ } \\
\text { pour les salariés concernés }\end{array}$ & $\begin{array}{c}\text { Nombre d'heures moyen dans le champ } \\
\text { pour les salariés concernés }\end{array}$ \\
\hline Spectacle vivant & 610 & 256 \\
\hline Architecture & 680 & 673 \\
\hline Conservation du patrimoine & 503 & 492 \\
\hline Activités cinématographiques et de vidéo & 578 & 255 \\
\hline Édition et librairie & 597 & 467 \\
\hline Presse & 737 & 498 \\
\hline Radio et télévision & 587 & 337 \\
\hline Ensemble & 508 & 367 \\
\hline
\end{tabular}




\begin{tabular}{|c|c|c|c|c|}
\hline \multirow[t]{2}{*}{ Secteurs } & \multicolumn{4}{|c|}{ Salaire annuel net } \\
\hline & Moyen & Médian & $10 \%$ & $90 \%$ \\
\hline Spectacle vivant & 5701 & 1571 & 125 & 16250 \\
\hline Architecture & 17025 & 15580 & 1570 & 32721 \\
\hline Conservation du patrimoine & 11836 & 8947 & 528 & 25465 \\
\hline Activités cinématographiques et de vidéo & 7999 & 1742 & 80 & 22571 \\
\hline Radio et télévision & 12445 & 3479 & 80 & 34925 \\
\hline Édition et librairie & 11676 & 7799 & 399 & 26021 \\
\hline Presse & 19246 & 15588 & 364 & 42377 \\
\hline Ensemble & 10511 & 4776 & 145 & 30212 \\
\hline
\end{tabular}

Lecture : le salaire annuel net moyen dans le spectacle vivant est de 5701 euros en $2006.50 \%$ de ces salariés ont perçu moins de 1571 euros (salaire médian). $10 \%$ des salariés ont perçu moins de 125 euros et $90 \%$ des salariés, plus de 16250 euros.

ainsi son maximum dans le spectacle vivant (écart de 1 à 3), les activités de radio et télévision (1 à 4) et celles de cinéma et vidéo ( 1 à 4 également), alors qu'il demeure, dans les autres secteurs, beaucoup plus modéré voire inexistant. La comparaison des deux courbes de concentration des revenus des secteurs de l'architecture et du spectacle vivant offre une bonne illustration de ces contrastes (graphique 4).

La très grande disparité des revenus tient donc, pour partie, au volume de travail moyen effectué par les salariés, très variable d'un secteur à l'autre. Cependant, d'importantes différences sectorielles subsistent lorsqu'on observe les salaires horaires des postes occupés dans chaque secteur (tableau 5). Le salaire horaire médian varie ainsi du simple au double: de 8,50 euros dans le secteur de l'édition et la librairie à 17 euros dans celui de la radio et télévision. Ce sont les différents secteurs du spectacle (activités cinématographiques et de vidéo, radio et télévision, spectacle vivant) qui connaissent les salaires horaires moyens les plus élevés, une caractéristique qu'il faut toutefois rapprocher de la très forte instabilité de l'emploi dans ces secteurs.

\section{Graphique 4 - Concentration comparée du revenu annuel des salariés dans l'architecture et dans le spectacle vivant}

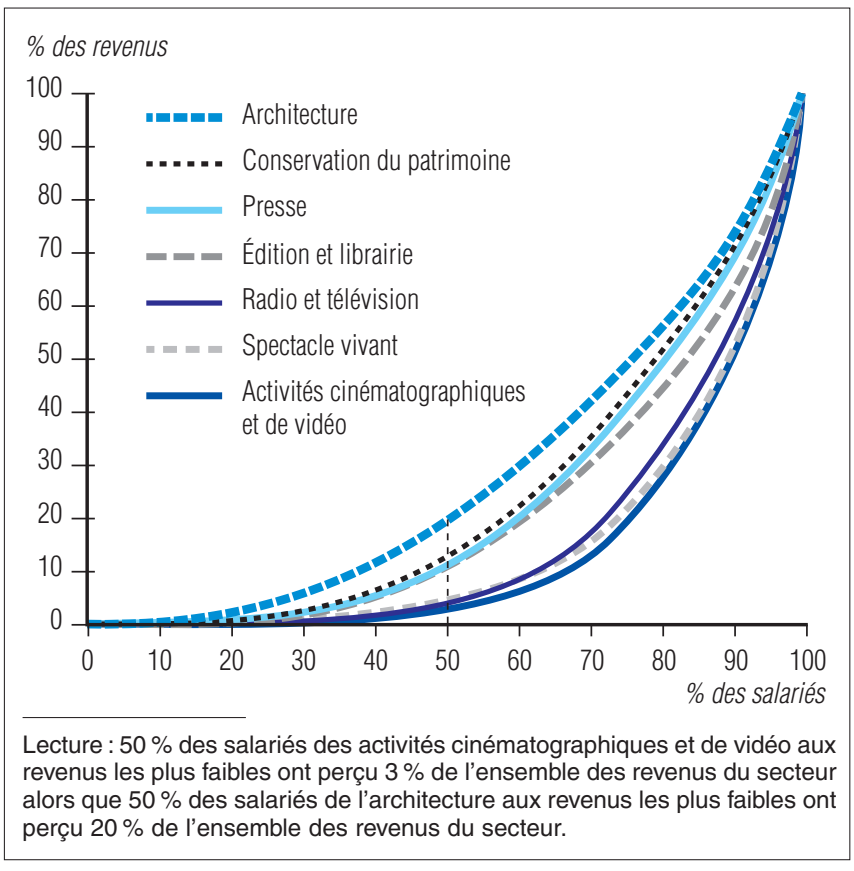

Source : DADS 2006, Insee/DEPS

Tableau 5 - Salaire net horaire selon l'activité en 2006

\begin{tabular}{lcccc} 
Secteurs & \multicolumn{3}{c}{ Salaire net horaire } \\
\cline { 2 - 4 } & Moyen & Médian & $\mathbf{1 0} \%$ & $\mathbf{9 0} \%$ \\
Spectacle vivant & 17,4 & 12,6 & 7,2 & 25,7 \\
Architecture & 16,0 & 11,0 & 7,1 & 19,4 \\
Conservation du patrimoine & 11,7 & 9,6 & 7,0 & 18,8 \\
Radio et télévision & 23,5 & 17,0 & 7,3 & 32,3 \\
Activités cinématographiques et de vidéo & 23,1 & 15,1 & 7,0 & 34,2 \\
Presse & 16,5 & 15,3 & 8,2 & 24,0 \\
Édition et librairie & 11,7 & 8,5 & 6,5 & $\mathbf{2 0 , 3}$ \\
Ensemble & $\mathbf{1 8 , 5}$ & $\mathbf{1 3 , 2}$ & $\mathbf{7 , 1}$ & $\mathbf{2 7 , 3}$
\end{tabular}

Lecture : dans le secteur du spectacle vivant, le salaire net horaire moyen est de 17,40 euros. $50 \%$ des salariés perçoivent un salaire horaire inférieur à 12,60 euros (salaire médian). $10 \%$ des salariés perçoivent un salaire horaire inférieur à 7,20 euros et $90 \%$ des salariés, un salaire horaire supérieur à 25,70 euros. 


\section{Un salarié sur deux n'était pas présent dans le secteur culturel l'année précédente}

$46 \%$ des salariés présents en 2006 dans le champ des activités culturelles n'étaient pas salariés de ce secteur en 2005. Une analyse récente, portant sur des années antérieures, réalisée à partir de la même source DADS ${ }^{7}$, semble confirmer, sur une plus longue période, l'ampleur de cette importante rotation (turn-over).

Ce taux varie selon l'activité considérée (graphique 5): en 2006, la part des entrants ${ }^{8}$ est ainsi de $30 \%$ dans les activités de presse, mais elle atteint $55 \%$ dans celles du cinéma et de la vidéo. Plus généralement, dans les activités de spectacle vivant, de cinéma et de vidéo, c'est un salarié sur deux (entre 48 et $55 \%$ ) qui « apparaît » en 2006.

Toutes activités confondues, l'âge médian des entrants - 32 ans - est de 7 ans inférieur à celui des salariés déjà présents en 2005 (39 ans). L'écart entre les deux sous-populations est moins marqué dans les activités du spectacle et de l'audiovisuel ( 3 ou 4 ans seulement), signalant une fois encore que l'accès au marché du travail, dans ces secteurs, est soumis à d'autres logiques que celles d'une stabilisation progressive au fil du cycle de vie professionnelle.

On observe à l'inverse des écarts plus conséquents (de 7 à 12 ans) dans les autres secteurs.

\section{Des entrants en sous-emploi}

De façon générale, les entrants en 2006 sont d'abord des personnes en sous-emploi : une majorité d'entre elles $(58 \%)$ a travaillé moins de 100 heures dans l'année, tandis que $9 \%$ seulement des salariés ont travaillé 1000 heures ou davantage (tableau 6). Plus globalement, la moitié de ces nouveaux salariés a travaillé moins de 60 heures dans l'année.
Graphique 5 - La part des entrants en 2006 selon l'activité

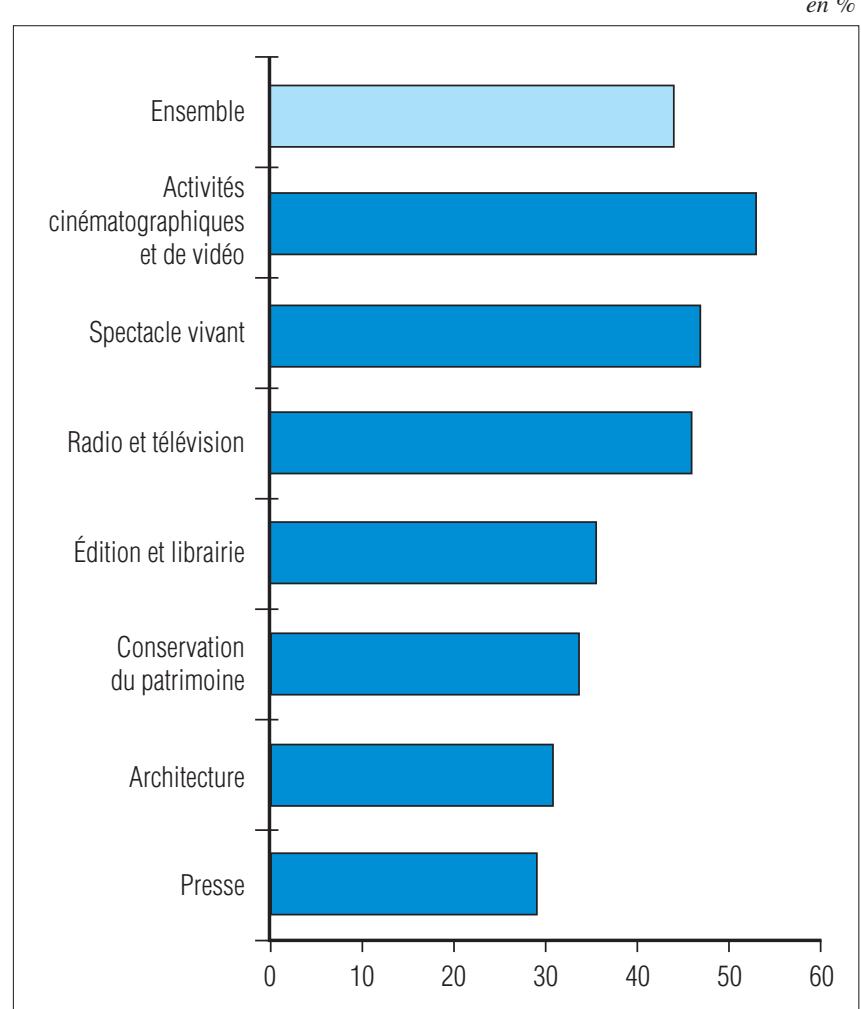

Lecture : $55 \%$ des salariés des activités cinématographiques et de vidéo repérés en 2006 n'étaient pas présents dans le secteur en 2005.

Source : DADS 2006, Insee/DEPS

Là encore, ce sont surtout les entrants des activités du spectacle et de l'audiovisuel qui sont concernés par ces très faibles volumes de travail: plus des deux tiers d'entre eux ont travaillé moins de 100 heures en 2006 et moins de $8 \%$, plus de 1000 heures. De ce fait, les disparités entre entrants et salariés déjà présents en 2005

Tableau 6 - Comparaison du temps de travail des entrants en 2006 et des salariés déjà présents en 2005, selon le secteur d'activité

\begin{tabular}{|c|c|c|c|c|}
\hline \multirow[t]{2}{*}{ Secteurs } & \multicolumn{2}{|c|}{ Entrants 2006} & \multicolumn{2}{|c|}{ Déjà présents en 2005} \\
\hline & de 100 heures & Plus de 1000 heures & Moins de 100 heures & Plus de 1000 heures \\
\hline Spectacle vivant & 67 & 5 & 29 & 23 \\
\hline Architecture & 12 & 30 & 2 & 69 \\
\hline Conservation du patrimoine & 26 & 19 & 10 & 65 \\
\hline Activités cinématographiques et de vidéo & 71 & 5 & 32 & 31 \\
\hline Radio et télévision & 67 & 8 & 28 & 42 \\
\hline Presse & 42 & 19 & 11 & 67 \\
\hline Édition et librairie & 31 & 19 & 14 & 62 \\
\hline Ensemble & 58 & 9 & 22 & 45 \\
\hline
\end{tabular}

7. Michel Amar, Malik KouBI, «Les entreprises du spectacle de 1995 à 2001 », Insee Première, no 978, juillet 2004.

8. Il s'agit ici uniquement des salariés de la culture présents en 2006 mais absents en 2005. Le terme d' «entrants » est employé ici par souci de simplification, une partie de ces salariés pouvant avoir été présents dans le secteur culturel en 2004 ou avant cette date et avoir connu une interruption temporaire d'activité en 2005. 
dans ces mêmes secteurs sont particulièrement marquées : dans le secteur des activités cinématographiques et de vidéo, la part des salariés travaillant plus de 1000 heures annuelles est six fois plus importante parmi les salariés déjà présents en 2005.

\section{Un risque de sortie élevé}

L'examen du devenir des salariés présents en 2005 confirme la grande vulnérabilité de ceux ayant peu travaillé au cours de l'année (tableau 7): une écrasante majorité des salariés ayant travaillé moins de 100 heures en $2005(83 \%)$ et $50 \%$ de ceux ayant travaillé entre 100 et 500 heures cette même année ne sont plus présents en 2006.
Cette observation qui désigne une catégorie de salariés particulièrement instable et peu insérée vaut pour tous les secteurs d'activité du champ culturel : ce sont toujours au moins les deux tiers des individus travaillant moins de 100 heures en 2005 qui se trouvent exclus l'année suivante (tableau 8).

Si le risque de sortie touche en priorité les salariés les moins insérés en 2005, il demeure non négligeable pour l'ensemble des salariés du champ, quelle que soit l'intensité de leur implication en 2005 - à l'exception de ceux travaillant l'équivalent d'un temps complet : par exemple, plus du tiers des salariés ayant effectué entre 500 et 1000 heures de travail en $2005(38 \%)$ ne sont plus présents l'année suivante.

Tableau 7 - Le devenir des salariés présents en 2005

\begin{tabular}{|c|c|c|c|c|}
\hline \multirow{2}{*}{$\begin{array}{l}\text { Nombre d'heures } \\
\text { de travail en } 2005\end{array}$} & \multicolumn{4}{|c|}{ Situation en 2006} \\
\hline & Amélioration ou stabilité* & Détérioration* & Sortie & Total \\
\hline Moins de 100 heures & 17 & - & 83 & 100 \\
\hline Entre 100 et 499 heures & 35 & 10 & 55 & 100 \\
\hline Entre 500 et 999 heures & 40 & 22 & 38 & 100 \\
\hline Entre 1000 et 1599 heures & 50 & 21 & 29 & 100 \\
\hline
\end{tabular}

\section{Tableau 8- Le devenir des salariés présents en 2005 selon le secteur d'activité}

\begin{tabular}{|c|c|c|}
\hline \multirow[t]{2}{*}{ Secteurs } & \multicolumn{2}{|c|}{ Moins de 100 heures en 2005} \\
\hline & Sortie en 2006 & Plus de 100 heures en 2006 \\
\hline Spectacle vivant & 78 & 5 \\
\hline Architecture & 72 & 14 \\
\hline Conservation du patrimoine & 71 & 8 \\
\hline Activités cinématographiques et de vidéo & 91 & 2 \\
\hline Radio et télévision & 87 & 3 \\
\hline Édition et librairie & 83 & 6 \\
\hline Presse & 74 & 10 \\
\hline
\end{tabular}

Source : DADS 2006, Insee/DEPS

Tableau récapitulatif - L'emploi salarié dans le secteur de la culture

\begin{tabular}{|c|c|c|c|c|c|c|c|}
\hline & $\begin{array}{c}\% \\
\text { des salariés } \\
\text { du secteur } \\
\text { culturel }\end{array}$ & $\begin{array}{l}\text { Nombre } \\
\text { d'heures } \\
\text { annuelles } \\
\text { travaillées } \\
\text { médian }\end{array}$ & $\begin{array}{c}\text { Salaire } \\
\text { annuel } \\
\text { net médian } \\
\text { (euros) }\end{array}$ & $\begin{array}{c}\% \\
\text { de salariés } \\
\text { en CDI }\end{array}$ & $\begin{array}{c}\% \\
\text { de salariés } \\
\text { à temps } \\
\text { complet }\end{array}$ & $\begin{array}{l}\text { \% de salariés } \\
\text { à avoir travaillé } \\
\text { dans un seul } \\
\text { établissement } \\
\text { au cours } \\
\text { de l'année }\end{array}$ & $\begin{array}{l}\text { \% des salariés } \\
\text { à avoir travaillé } \\
\text { aussi hors } \\
\text { champ culturel }\end{array}$ \\
\hline Spectacle vivant & 34 & 125 & 1571 & 22 & 40 & 77 & 37 \\
\hline Architecture & 6 & 1575 & 15580 & 76 & 74 & 95 & 19 \\
\hline Conservation du patrimoine & 4 & 991 & 8947 & 38 & 59 & 98 & 24 \\
\hline $\begin{array}{l}\text { Activités cinématographiques } \\
\text { et de vidéo }\end{array}$ & es 16 & 104 & 1742 & 24 & 50 & 81 & 27 \\
\hline Radio et télévision & 15 & 197 & 3479 & 29 & 53 & 81 & 25 \\
\hline Édition et librairie & 11 & 914 & 7799 & 68 & 62 & 95 & 26 \\
\hline Presse & 14 & 1274 & 15588 & 64 & 66 & 88 & 23 \\
\hline
\end{tabular}




\section{Quelques caractéristiques des établissements de la culture}

En 2006, 65000 établissements du secteur de la culture (voir définition du champ dans l'encadré méthodologique) ont employé du personnel salarié. $31 \%$ de ces établissements sont situés en île-deFrance. Ils représentent $51 \%$ du volume horaire travaillé. La part des établissements franciliens varie fortement selon le secteur d'activité : $15 \%$ d'établissements franciliens dans la conservation du patrimoine, mais $59 \%$ dans les activités cinématographiques et de vidéo, pour une part du volume horaire respective de $61 \%$ et $72 \%$.

Le secteur est composé essentiellement d'établissements de petite taille: 6 salariés en moyenne, variable ici encore selon le secteur ( 3 salariés en moyenne dans l'architecture, 18 en moyenne dans les établissements de la radio et télévision).

$44 \%$ des établissements de la culture sont des sociétés, $34 \%$ des associations, $22 \%$ des entreprises individuelles.

\section{Portrait sociodémographique des salariés du secteur culturel}

Les salariés de la culture ont en moyenne 37 ans. D'un secteur d'activité à l'autre, l'amplitude des âges moyens et médians est peu importante, de l'ordre de quatre ans en moyenne. Ce sont les salariés du spectacle vivant et des activités cinématographiques et de vidéo qui sont les plus jeunes (la moitié d'entre eux a moins de 34 ans et trois sur quatre, moins de 44 ans), mais la différence avec les autres secteurs reste modérée.

La répartition par sexe est extrêmement contrastée selon le secteur. Dans les activités du spectacle vivant et de l'audiovisuel, les hommes sont très fortement sur-représentés $(61 \%$ dans le cinéma et la vidéo, $60 \%$ dans la radio et télévision, $58 \%$ dans le spectacle vivant). Dans l'architecture, la conservation du patrimoine, l'édition et la librairie, au contraire, ce sont les femmes qui sont majoritaires (respectivement $53 \%, 57 \%$ et $61 \%$ ). La presse est le seul secteur au sein duquel s'observe une parité parfaite entre les salariés des deux sexes.

Les salariés des secteurs du cinéma et de la vidéo, ceux de la radio et télévision travaillent très majoritairement en Île-de-France (deux salariés sur trois). Cette concentration géographique francilienne s'observe également, bien que dans une moindre mesure, chez les salariés de la conservation du patrimoine, de la presse, de l'édition et librairie (respectivement $55 \%, 49 \%$ et $42 \%$ de Franciliens). Quant aux salariés de l'architecture et du spectacle vivant, ils travaillent en revanche très majoritairement au sein d'établissements non franciliens (respectivement $72 \%$ et $63 \%)$.

\section{Méthodologie}

\section{La source DADS}

Les données présentées dans cette note proviennent d'une exploitation des Déclarations annuelles de données sociales (DADS) de I'Insee. Les DADS sont une formalité administrative obligatoire pour toute entreprise employant des salariés. Elles permettent d'élaborer à un rythme annuel des statistiques sur l'emploi et les salaires en France. Deux millions d'établissements remplissent chaque année cette déclaration, représentant plus de 50 millions de lignes salaires. Les DADS couvrent environ $80 \%$ de la population salariée totale (quelques catégories de salariés sont en effet exclues du champ de l'exploitation: les agents des organismes de l'État, les salariés des services domestiques et ceux des activités extra-territoriales). Les DADS contiennent des informations sur l'établissement employeur, sa localisation, ses salariés, les caractéristiques des emplois et les rémunérations correspondantes.

\section{Quelques définitions}

- Établissement: l'établissement est une unité de production géographiquement individualisée, mais juridiquement dépendante d'une entreprise.

- Poste : un poste correspond au cumul des lignes "salarié » d'un même salarié dans un même établissement. Il est identifié par le couple individu/Siret. Ainsi, un salarié ayant travaillé dans deux établissements différents au cours de l'année se retrouve dans deux postes différents.

- Période : un salarié peut avoir plusieurs périodes d'emploi dans un même établissement. La notion de période recouvre plusieurs cas de figure : plusieurs contrats à l'intérieur d'un même établissement, mais aussi changement de caractéristiques d'un poste. Cette variable retranscrit donc imparfaitement la multiplication des contrats mais donne cependant une bonne indication sur le sujet.

\section{Le champ retenu}

L'étude a privilégié une approche sectorielle du champ de la culture et non une approche par professions.
Le champ "culture " est défini à partir de l'activité de l'établissement employeur, par référence aux codes de la nomenclature d'activités française (Naf) suivants:

Édition et librairie : $22.1 \mathrm{~A}$ édition de livres, 22.1G édition d'enregistrements sonores, 22.1J autres activités d'édition, 52.4R commerce de détail de livres, journaux, papeterie ;

Presse : $22.1 \mathrm{C}$ édition de journaux, 22.1E édition de revues et périodiques, $92.4 Z$ agences de presse, journalistes indépendants;

Radio et télévision : $92.1 \mathrm{~A}$ production de films pour la télévision, 92.2A activités de radio, 92.2B production de programmes de télévision, 92.2D édition de chaînes généralistes, 92.2E édition de chaînes thématiques et $92.2 \mathrm{~F}$ distribution de bouquets de programmes de télévision;

Activités cinématographiques et de vidéo : $92.1 \mathrm{~B}$ production de films institutionnels et publicitaires, $92.1 \mathrm{C}$ production de films pour le cinéma, 92.1D prestations techniques pour le cinéma et la télévision, 92.1F distribution de films cinématographiques, 92.1G édition et distribution vidéo et $92.1 \mathrm{~J}$ projections de films cinématographiques ;

Architecture : $74.2 \mathrm{~A}$ activités d'architecture;

Spectacle vivant et activités artistiques : 92.3A activités artistiques, 92.3B services annexes aux spectacles, 92.3D gestion de salles de spectacles, 92.3K activités diverses du spectacle;

Conservation du patrimoine : $92.5 \mathrm{~A}$ gestion des bibliothèques, 92.5C gestion du patrimoine culturel.

Le champ « culture » ainsi défini, tous les salariés ayant travaillé au moins une heure dans ce champ en 2006 ont été retenus pour l'exploitation statistique. Pour chacun de ces salariés, l'ensemble des périodes de travail hors champ a été pris en compte, afin de pouvoir reconstituer l'ensemble des heures travaillées sur l'année.

Rappelons, enfin, que la source exploitée ici ne couvre que l'emploi salarié du secteur culturel - l'emploi non salarié représentant environ un quart de l'emploi total de ce secteur*.

* D'après l'enquête Emploi de l'Insee. Voir Éric Cléron, Frédérique Patureau, l'Emploi dans le secteur culturel en 2005, Paris, DEPS, Ministère de la Culture et de la Communication, coll. «culture chiffres », 2007-7. 